\title{
LOS EGRESADOS UNIVERSITARIOS Y LA INSERCIÓN LABORAL: UN ACERCAMIENTO AL PANORAMA LATINOAMERICANO Y ESPAÑOL
}

\author{
University graduates and placement: an approach to the Latin American and Spanish \\ panorama
}

Maria Luisa Solé-Moro iD

PhD Business. Universidad de Barcelona, España, mlsolesole@ub.edu

Javier A. Sánchez-Torres ic

PhD Business. Universidad de Medellín, Colombia, jasanchez@udem.edu.co

Francisco-Javier Arroyo-Cañada

PhD Business. Universidad de Barcelona, España, fjarroyo@ub.edu

\section{Ana Argila-Irurita}

PhD Business. Universidad de Barcelona, España, aargila@ub.edu

\begin{abstract}
Cómo citar / How to cite
Solé-Moro, M. L., Sánchez-Torres, J., Arroyo-Cañada, F. J., \& Argila-Irurita, A. (2018). Los egresados universitarios y la inserción laboral: un acercamiento al panorama latinoamericano y español. Revista CEA, 4(8), 67-74. https://doi.org/10.22430/24223182.1048
\end{abstract}

Recibido: 10 de febrero de 2018

Aceptado: 26 de mayo de 2018

\section{Resumen}

Este artículo se presenta como una reflexión, a partir de una revisión de la literatura sobre la inserción laboral del graduado universitario en España y Latinoamérica; el panorama de globalización, volatilidad económica, aumento de graduados universitarios y otros fenómenos generan dificultad en el acceso al mercado laboral de quienes terminan su paso por la universidad, desde los estudios de segundo ciclo (carrera profesional) hasta tercer ciclo de formación (postgrados).

Los resultados encontraron que existen pocos estudios en el área, a pesar de que es en los últimos años donde se han desarrollado, especialmente a finales de la década pasada, así mismo, se detectaron estudios respecto al egresado y a su empleabilidad, analizando básicamente datos estadísticos con los ingresos (tipo de empleo y salario) y, por otra parte, otros estudios que buscan relaciones con la reorganización y los cambios en la educación superior.

Como una de las conclusiones más importantes, se tiene que los estudios sobre el tema han detectado las debilidades y fortalezas del recién graduado, así como otros han permitido conocer los requerimientos y puntos de atención en la formación específica que buscan las empresas.

Palabras clave: empresa, graduado, inserción laboral, universidad, política educativa.

\section{Abstract}

A panorama of globalization, economic volatility, increasing numbers of university 
graduates, and other phenomena restrict the access of university graduates (with bachelor's or graduate degrees) to the labor market. This article presents a reflection on graduate placement research in Spain and Latin America.

The results of this literature review suggest there is a limited number of studies in the field. Nevertheless, most of them have been conducted in recent years, especially at the end of the last decade. This review also identified articles about graduates and employability that basically analyze statistical data along with income (type of employment and salary). Other authors have examined relationships with reorganization and changes in higher education.

Finally, while part of the studies on the subject have detected the weaknesses and strengths of the recently graduated, others have shed light on requirements and points of attention regarding the specific type of education that companies require. This insight is invaluable for developing educational policies that address the needs of the current labor market.

Keywords: company, graduate, work placement, university, educational policy.

\section{INTRODUCCIÓN}

El desarrollo de la sociedad y del conocimiento depende de la producción de nuevos conocimiento y de su transmisión a través de la educación, la formación y la divulgación de su uso a través de la universidad, teniendo como misión fundamental la de proporcionar una formación y cualificaciones suficiente que permita la empleabilidad y competitividad de sus egresados (Martín Del Peso et al., 2013).

Por tanto, el acceso del egresado universitario al mercado laboral, ha sido un asunto de gran importancia en la última década para las universidades, debido a que estas han tenido un elevado crecimiento desde finales del siglo XX y la primera década del siglo XXI, esta constante ha generado que las universidades tengan que rendir informes sobre su gestión y resultados; a partir de esto, se crean investigaciones realizadas por las mismas universidades, por ejemplo, en Estados Unidos, un $70 \%$ de los estudios enfatiza en el aspecto socioeconómico, mientras que solo un $15 \%$ se enfocó en el aprendizaje y otro $15 \%$ a las contribuciones del egresado a su universidad (de Vries et al., 2013).

Así mismo, se han generado grandes limitantes en la investigación sobre la inclusión laboral del egresado universitario, ya que, como lo explica de Vries et al. (2013), las universidades realizan estudios propios con metodologías diversas que no permiten hacer estudios comparativos o de mayores dimensiones, quedándose en lo particular; así mismo, la educación superior ha sido un terrenos de grandes reformas lo que impacta en las políticas y su continuidad respecto al éxito laboral del recién graduado.

Según (Espinar et al., 2010), solo hasta la primera década de este siglo se puso de manifiesto la necesidad de analizar la situación del empleo de los graduados universitarios, surgen iniciativas de las asociaciones de universidades, en España ANECA propone que se realice un seguimiento anual a los egresados para conocer su situación laboral. La rendición de cuentas a la que está sujeta la universidad ante la sociedad ha llevado a que se tome como indicador de calidad la eficaz inserción del graduado en el mercado laboral; por otro lado, surge la discusión sobre la razón de ser de la académica, respecto a que no se puede convertir en un proveedor de titulados operativos, sino una formación centrada en el estudiante como un individuo social.

Surgen dos líneas de investigación respecto al egresado y su empleabilidad; la primera relacionada básicamente con los ingresos (tipo de empleo y salario), y por otra parte, 
otros estudios buscan relaciones con la reorganización y los cambios en la educación superior; para de Vries et al. (2013) rara vez se interconectan, con lo cual los estudios sobre el mercado laboral no analizan los factores de preparación como posible explicación, por su parte, los estudios sobre las universidades y las reformas implementadas no analizan cuales son los efectos de estos en el mercado laboral.

Todo lo anterior, ligado a un contexto de creciente problemática del paro profesional y del empleo precario de la última década, requiere analizar de manera sistémica y rigurosa acerca de los procesos de inserción socio laboral de los titulados universitarios, tanto para la mejora de la educación como para dar respuesta a las demandas sociales y laborales (Vivas, 2009).

El objetivo de este artículo es, por tanto, examinar a partir de una revisión bibliográfica, cuáles son los aspectos que determinan la efectiva inserción laboral del egresado universitario y los aspectos más importantes para alcanzarla.

Se ha estructurado el trabajo de la siguiente manera: en primer lugar, se plantea una revisión bibliográfica de estudios específicos sobre la temática; en segundo lugar, se plantean a partir de los resultados de la revisión literaria, los aspectos que las universidades deben tener en cuenta para la efectiva colocación del graduado en el mercado laboral.

\section{METOdOLOGÍA}

Se realizó una investigación basada en una revisión de la literatura con enfoque descriptivo, tomando en cuenta la escasa información que se encuentra en las bases de datos oficiales de los estamentos oficiales, como Ministerios de Educación, organizaciones universitarias y otros organismos relacionados con educación superior.

Después de aplicar los criterios en las bases de datos (Tabla 1), los resultados fueron escasos, teniendo como resultado en su mayoría estudios enfocados a problemáticas especificas 0 casos sobre tópicos relacionados con las palabras clave, pero que no responden al panorama en general o que han perdido pertinencia por no ser recientes, teniendo por tanto que para el intervalo de tiempo filtrado (2000-2018) se obtuvieron un total de 14 artículos empíricos.

Tabla 1. Criterios de la revisión bibliográfica

Table 1. Criteria for the literature review

\begin{tabular}{l|l}
\hline Bases de datos & Scopus, Web of Science, Ebsco, Google Academic \\
fecha & Enero - mayo 2018 \\
Palabras clave & educación, universidad, egresado \\
filtros & Geográfico ( Latinoamérica y España) temporal (2000-2018). \\
\hline \multicolumn{1}{l}{ Fuente: elaboración propia }
\end{tabular}

\section{RESULTADOS}

Los hallazgos presentan que en su mayoría los estudios se han realizado en España, así mismo la el principal enfoque en las metodologías de investigación es el uso de bases de datos sobre tasas de empleo y enfocado a los egresados, empresas y universidades; como lo muestra el estudio de
García \& Díaz (2009), en el que se desarrolló un estudio de carácter descriptivo utilizando metodologías de cruce de datos entre Universidad y Servicio Público de Empleo. Sin embargo, según Martin del Peso (2013), este tipo de metodologías no tienen en cuenta a todos los actores, lo que genera grandes sesgos de información y un reflejo parcial de la realidad. 
Otro gran problema que describe GarcíaEspejo e Ibáñez-Pascual (2006), es la enorme variedad de titulaciones convierte en inadecuada la tendencia a utilizar análisis generalistas, ya que distorsionan los resultados obtenidos al ocultar particularidades propias de cada carrera universitaria. Así mismo, recomienda que el estudio de competencias para el empleo se realice a partir de los puestos de trabajo que reconozcan y exijan el nivel universitario de quien los ocupa, estas serán las competencias que deben ser atendidas por el sistema educativo.

También se encontraron enfoques más precisos que buscaron examinar cuales son las competencias de empleabilidad de los egresados universitarios requeridas por el mercado laboral (García Espejo and Ibáñez Pascual, 2006), donde se analizan diferentes variables relacionadas con la empleabilidad, la transparencia y la comparabilidad; para este estudio en particular se analizó el ajuste de competencias a partir de la relación entre currículo educativo y los perfiles profesionales requeridos por el mercado.

Según Martín del Peso (2013) la velocidad con la que los graduandos aprenden a desarrollar su trabajo depende del nivel y del tipo de competencia adquirida en su paso por la universidad; siendo necesario definir un perfil profesional que determine cuáles son las competencias que debe reunir un titulado a lo largo de su formación universitaria, teniendo preciso contar con los grupos de interés internos (la sociedad académica) y los grupos de interés externos (los empleadores, empresas, gobierno).

A partir de esto, Martín del Peso (2013) realizó un estudio con 564 empleadores en la Comunidad de Madrid-España, indagando sobre tres grupos de competencias (instrumentales, interpersonales y sistémicas) además de las específicas y genéricas para graduados de ingenierías; los resultados destacaron en que los egresados no se ajusta al nivel demandado por las empresas, específicamente, las competencias interpersonales presentan un desajuste alto entre lo que espera la empresa y las habilidades del graduado; siguen las competencias específicas y genéricas, mientras que las competencias sistémicas (capacidades creativas, reflexivas y de generar conocimientos) son las que mejor se adecuan a las necesidades de los empresarios. Así mismo, los empresarios definieron al titulado ideal como aquel que tiene conocimientos básicos y solidos de su titulación, que posea un cierto conocimiento práctico (resultado de prácticas laborales durante sus estudios), que domine el idioma ingles y que incorpore motivación y capacidad para el aprendizaje.

Validan estos supuestos para el caso español, los resultados anteriores del estudio desarrollado por Pol et al. (2009), realizado a 500 empresas y universidades de las Islas Baleares, en el cual se resalta la importancia de las competencias genéricas en el egresado (conocimientos específicos del área de estudio), así como también, la necesidad de desarrollar las competencias sistémicas (habilidades y actitudes personales y laborales) durante los estudios universitarios; al igual que como concluye García-Blanco \& Cárdenas Sempértegui (2018) en su estudio sobre la inserción laboral en Latinoamérica, concluyendo que, la incorporación de competencias claves en el estudiante universitario le permite una mejor inserción laboral.

Existen varios estudios que se han enfocado en conocer el contexto social, familiar, educativo y cultural del recién graduado y sus relaciones con el acceso al mercado laboral (García-Espejo \& Ibáñez-Pascual, 2006; de Vries et al., 2008, 2009; Flores et al., 2009; Martín-Del Peso, Rabadán Gómez and Hernández-March, 2013). El contexto socioeconómico es un factor fundamental en el éxito laboral del recién graduado, demostrándose en el estudio de de Vries et al. (2013) realizado a más de 50 instituciones 
universitarias latinoamericanas, que un factor influyente en el éxito profesional, es el nivel de estudios de los padres del estudiante, mientras mayor sea este, mayor será el ingreso mensual que obtiene el egresado en su salario, es decir, mejores cargos en el mercado laboral; así mismo, se resalta que la experiencia laboral previa a recibir el título universitario también influye en obtener mejores trabajos cuando terminan los estudios; el factor género, continua siendo moderador, los hombres continúan recibiendo un salario más alto que las mujeres; otro factor influyente es el tipo de universidad, los egresados de universidades privadas ganan hasta el doble de los que salen de las públicas, incluso se generan excepciones en las diferencias generales sobre el género, teniendo que las mujeres graduadas de universidades privadas ganan más que los hombres egresados de las públicas. Finalmente, para este estudio, se resalta que los graduados consideran que las capacidades que permiten el acceso al mercado laboral y a mejores condiciones son las competencias sistémicas (como aplicar los conocimientos a la práctica o la toma de decisiones), así mismo, el dominio de un segundo idioma tiene relación con el triunfo laboral, pese a que quienes han realizado estancias en el extranjero reportaron ingresos más bajos.

Por su parte, la tipología de los estudios también influye directamente en el futuro laboral, ya que las últimas estadísticas del Ministerio de Educación de España (MDECE and CCS, 2015) muestran que los egresados españoles de carreras profesionales del ámbito de la salud y veterinaria son los que más encuentran trabajo después de graduarse, mientras que los graduados que tienen menores posibilidades de encontrar trabajo son las titulaciones de Turismo, Administración y Negocios, Artes y Periodismo y Comunicación (Figura 1); sin embargo, la dinámica de este factor es diferente en cada contexto o país iberoamericano y solo debe tenerse en cuenta para el análisis de cada caso en particular.

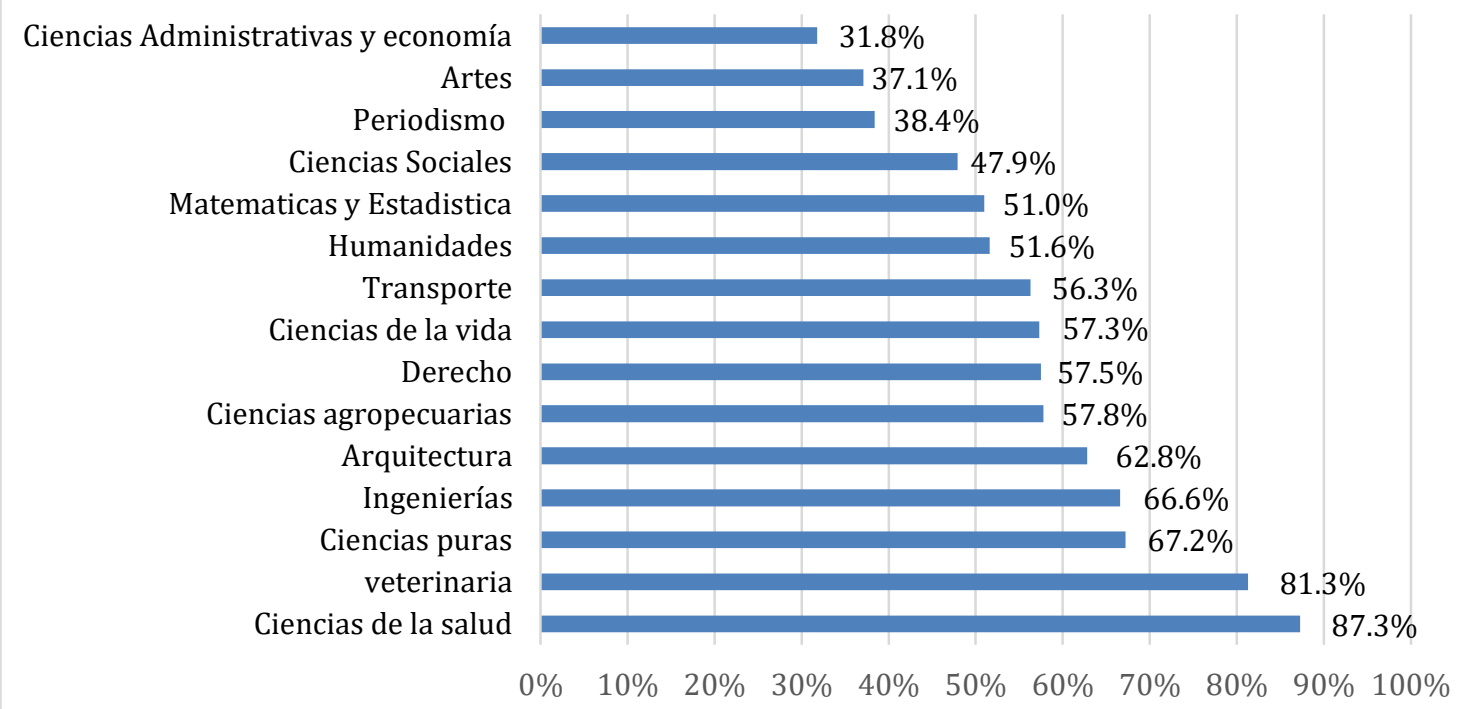

Figura 1. Porcentaje egresados universitarios en el grupo de cotización de la seguridad social después de finalizar sus estudios por ámbito de estudio

Figure 1. Percentage of university graduates that pay social security grouped by field Fuente: Adaptado de (MDECE and CCS, 2015). 
En esta línea de investigación, otro factor determinante es el expediente académico, los resultados de García \& Díaz (2009) demuestran que en la mayoría de las carreras universitarias, las notas de los egresados se relacionan de manera inversamente con la obtención de un empleo, teniendo que aquellos egresados con mayores notas tardan más tiempo en obtener un primer empleo, siendo mayor esta significancia en titulaciones como Biología, Ciencias Químicas - Psicología; mientras que para Medicina y Fisioterapia los resultados fueron contrarios, teniendo que quienes se insertan en el mercado laboral primero son quienes obtuvieron notas más altas.

Otro estudio realizado en España analizó la relación entre la nota medio de egreso y el tiempo que se tarda en enrolarse en el mercado laboral y en la continuidad de estudios del tercer ciclo (postgrados, master y doctorado) (De Vries, Vázquez-Cabrera \& Ríos-Treto, 2013), los resultados confirman que existe una relación positiva entre las calificaciones notables y el que los graduados continúen estudios de postgrado, mientras que para el caso de la relación entre calificaciones y acceso al trabajo, se tiene que a mejores notas más difícil es el acceso al mercado laboral, especialmente para titulaciones como las ciencias puras y las ciencias sociales. Solo para las carreras de las ciencias de la salud, por resultados, se comportan de manera lógica y quienes tienen mejores notas durante su carrera universitaria se incorporan más rápido y con mejores condiciones laborales.

Un factor que resaltan algunos estudios sobre los egresados universitarios es la sobrecualificación. Para el estudio de Montalvo (2009) también en España, demuestra que los niveles de sobre cualificación del profesional español continúa siendo muy elevado incluso cinco años después de graduarse los cuales generan condiciones laborales por debajo de los conocimientos y titulaciones adquiridas, aunque el estudio de Pastor \& Peraita (2014) contradiciendo estos resultados, al encontrar que, a mayor cualificación mejores condiciones laborales.

En cuanto a la movilidad de los profesionales, Montalvo (2009) comprobó que los factores geográficos y culturales afectan negativamente el acceso al mercado, debido a que se presentan baja flexibilidad tanto geográfica como funcional de los trabajadores.

\section{CONCLUSIONES}

Concluyendo este análisis sobre la investigación en la inserción del egresado universitario en el mercado laboral, se encontró que este presenta una evolución desde la última década del siglo $\mathrm{XX}$, suceso que puede estar ligado a los crecientes procesos de acreditación y evaluación de la calidad de las universidades. Sin embargo, los estudios se han conducido sesgadamente en la mayoría de los casos, ya que analizan individualmente a los agentes involucrados (egresados, sector empresarial y universidad), o también los estudios se enfocan únicamente en determinar las capacidades y aspectos de formación óptimos, dejando de lado la propuesta de soluciones prácticas y aplicables a la mejora de la problemática que crece en muchas de las profesiones en España y Latinoamérica, como afirma (García-Blanco \& Cárdenas Sempértegui, 2018), es necesario por tanto potenciar estudios que generen información y herramientas que permitan el fortalecimiento de las políticas universitarias, para solventar los problemas de la inserción laboral del universitario.

Así mismo, los resultados demostraron que la universidad es el actor fundamental de todo el proceso; la universidad debe establecer estrategias de aprendizaje y refuerzo de todas las capacidades y habilidades transversales a los planes curriculares necesarios para el triunfo laboral del estudiante, proponiendo, por ejemplo, 
reformulación de los programas formativos (Espinar et al., 2010), ya que tiene la capacidad y pertinencia de intervenir en los estudiantes otorgándoles finalmente todas las herramientas que les permiten tener oportunidades laborales con equidad (Fachelli, Torrents and Navarro-Cendejas, 2014). Para este fin, las universidades deben examinar cada programa y adecuar las necesidades que demandan los empleadores y estudiantes, con el objetivo de alinear esa brecha que se genera entre mercado laboral y profesionales recién graduados; estas acciones deben tener en cuenta estudios longitudinales que permitan detectar realmente cuáles son las falencias en capacidades, metodologías y programas educativos que requieren una intervención desde la planificación curricular de la alma mater.

Las limitaciones de este estudio radicaron en la escasa obtención de estudios empíricos, especialmente recientes, factor que no permitió realizar mayores análisis de la información, lo que genera proponer que se realicen en el futuro estudios completos sobre esta problemática y los procesos desde las universidades, como el seguimiento a los graduados, la extensión universitaria con la empresa y la sociedad, los procesos de mejora curricular y la formación integral.

\section{REFERENCIAS}

Espinar, S. R., Nebot, A. P., Arjona, L. B., \& Castiñeira, S. S. (2010). Sobre la empleabilidad de los graduados universitarios en Catalunya: del diagnóstico a la acción Graduate employability in Catalonia. From diagnosis to action. Revista de Educación, 351, 107-137.

Fachelli, S., Torrents, D. \& Navarro-Cendejas, J. (2014). La universidad española suaviza las diferencias de clase en la inserción laboral. Revista de Educación, 364, 119-144.
Gil-Flores, J., García Jiménez, E., \& Santos López, C. (2009). Miradas retrospectivas de los egresados sobre la educación superior. Revista de Investigación Educativa, 27( 2), 371-393.

García, L. A., \& Díaz, C. E. (2009). Relaciones entre la nota de egreso de los titulados universitarios y su inserción laboral. European Journal of Education and Psychology, 2(3), 169-180.

García-Espejo, I. \& Ibáñez-Pascual, M. (2006). Competencias para el empleo. Demandas de las empresas y medición de los desajustes. Revista Internacional de Sociología, 44 (43), 139-168.

García-Blanco, M. \& Cárdenas Sempértegui, E. B. (2018). La inserción laboral en la Educación Superior. La perspectiva latinoamericana. Educación XX1, 21(2), 323-347. doi: 10.5944/educxx1.16209.

Martín-Del Peso, M., Rabadán-Gómez, A. B. \& Hernández-March, J. (2013). Desajustes entre formación y empleo en el ámbito de las enseñanzas técnicas universitarias: La visión de los empleadores de la Comunidad de Madrid. Revista de Educación, 360, 244-267.

MDECE and CCS (2015) Inserción laboral de los egresados universitarios. La perspectiva de la afiliación a la Seguridad Social. Primer informe. Spain.

Montalvo, J. (2009). La inserción laboral de los universitarios y el fenómeno de la sobrecualificación en España. Papeles de Economía Española,119, 1-29.

Pastor, J. M. \& Peraita, C. (2014). La inserción laboral de los universitarios españoles. Revistade la Asociación de Sociología de la Educación (RASE), 7(1), 367-372.

Pol, A. P., Moreno, J. J. M. \& Oliver, M. P. (2009). Las competencias genéricas en 
la educación superior. Estudio comparativo entre la opinión de empleadores y académicos. Psicothema, 21(3), 433-438.

Vivas, A. J. (2009). Reflexiones sobre la necesidad de acercamiento entre universidad y mercado laboral. Revista Iberoamericana de Educación, 50, 1-8.

De Vries, W., Cabrera, A., Vázquez, J., \& Queen, J. (2008). Conclusiones a contrapelo. La aportación de distintas carreras universitarias a la satisfacción en el empleo. Revista de la Educación Superior, 37(146), 67-

De Vries, W., Vázquez-Cabrera, R., \& RíosTreto, D. (2013). Millonarios o malparados: ¿de qué depende el éxito de los egresados universitarios? Revista Iberoamericana de Educación Superior, 4(9), 3-20. 